\title{
Adsorption et transfert de matière en ultrafiltration
}

\author{
par \\ Sémia BAKLOUTI, P. AIMAR, V. SANCHEZ*
}

\section{Rés u m é}

Le transfert de matière dans une unité d'ultrafiltration dépend de la nature de la membrane, de la nature de la solution et des conditions opératoires.

Des recherches récentes montrent que dans le cas de solutés protéiques, l'adsorption sur la membrane joue un rôle important sur le transfert de solvant.

Cet effet est étudié ici dans le cas de la sérum albumine bovine et de membrane IRIS 3038 ; on déterminera ainsi une loi de variation de la résistance hydraulique en fonction du temps et de la concentration.

Les courbes expérimentales d'ultrafiltration de solutions protéiques peuvent alors être interprétées en combinant la théorie du film, l'effet dû à la pression osmotique et en tenant compte de cette cinétique d'adsorption.

Mots clés : Ultrafiltration - Adsorption - Albumine.

\section{INTRODUCTION}

En ultrafiltration, le transfert de matière au travers de la membrane dépend de nombreux paramètres tels que :

- la nature de la membrane;

- la nature de la solution à ultrafiltrer ;

- les conditions opératoires : vitesse de circulation $\mathbf{u}_{0}$, différence de pression appliquée $\Delta \mathrm{P}$, géométrie de l'appareil.

* Laboratoire de Chimie-physique et électrochimie (L.A. 192), Université PaulSabatier, 118, route de Narbonne - 31062 Toulouse cedex. 
Plusieurs théories ont été proposées pour décrire les phénomènes observés :

- la théorie du film fait appel à la formation d'une couche de gel à la membrane pour expliquer l'existence d'un flux limite de solvant;

- dans le cas de la théorie de la pression osmotique, l'augmentation de concentration à la membrane $\mathrm{C}_{\mathrm{m}}$, due à la polarisation entraîne une augmentation de pression osmotique $\Delta \pi$, qui peut être du même ordre de grandeur que l'accroissement de pression appliquée ;

- la théorie de la résistance à la filtration fait intervenir l'accroissement de la quantité de matière accumulée à la membrane pour justifier la limitation du flux de solvant.

Chacune de ces théories, prise de façon indépendante, décrit les résultats expérimentaux de manière qualitative ou quantitative dans certains cas particuliers. Ceci conduit à s'interroger, comme le font certains auteurs (Trettin et Doshi, 1981), sur l'importance relative de chacun des phénomènes qu'elles décrivent. D'autre part, aucune d'elles ne tient compte de l'adsorption des solutés sur les membranes. Or, des travaux récents (Fane et coll., 1983; Howell et coll., 1981; Ingham et coll., 1980; Kozinski et Lightfoot, 1972) montrent que l'adsorption peut réduire de façon considérable (jusqu'à $50 \%$ ) le flux de perméation de l'eau et ceci même pour des concentrations très faibles en soluté protéique. Fane et coll, en 1983, ont ainsi attribué, dans le cas de la sérum albumine bovine, BSA, la limitation du flux de perméation à l'augmentation de la quantité de protéine adsorbée en cours de fonctionnement.

Dans ce travail, on étudie l'adsorption de la BSA sur des membranes polymères et son influence sur le transfert de matière. A partir des résultats expérimentaux, on établit une loi cinétique reliant la résistance apparente hydraulique de la membrane à la concentration et au temps. Cette loi, utilisée conjointement avec les théories du film et de la pression osmotique, permet d'interpréter les courbes expérimentales reliant la vitesse de transfert $\mathrm{V}$, à la différence de pression appliquée et ce, pour diverses concentrations initiales $C_{0}$, en BSA.

\section{THEORIE}

Le phénomène de polarisation à l'interface membrane-solution est le plus souvent caractérisé par le modèle de la théorie du film. En régime permanent et en un point donné de la membrane, l'apport de matière par convection est compensé par un flux de diffusion en sens inverse. En ce point, la vitesse de filtration est donnée par la relation :

$$
\mathrm{V}=\mathrm{k} \cdot \ln \left(\mathrm{C}_{\mathrm{m}} / \mathrm{C}_{0}\right)
$$


$\mathrm{k}=\mathrm{D} / \delta$ est le coefficient de transfert de matière à l'interface, $\mathrm{D}$ est le coefficient de diffusion du soluté à la membrane, $\delta$ est l'épaisseur de la couche de polarisation.

Pour expliquer les courbes $\mathrm{V}=\mathrm{f}(\Delta \mathrm{P})$ où l'on voit que $\mathrm{V}$ devient plus ou moins rapidement indépendant de $\Delta \mathrm{P}$ pour atteindre une valeur limite $V_{1 i m}$, de nombreux auteurs depuis Blatt et coll. en 1970, supposent que la concentration à la membrane est alors devenue suffisante pour que se forme une couche de gel, de concentration $\mathrm{Cg}$.

Dès lors, en supposant que le coefficient de transfert $\mathrm{k}$ est indépendant de $\mathrm{C}$ et de $\Delta \mathrm{P}$, on obtient, pour un régime hydrodynamique donné :

$$
\mathrm{V}_{\mathrm{itm}}=\mathrm{k} \cdot \ln \left(\mathrm{C}_{\mathrm{g}} / \mathrm{C}_{0}\right)
$$

Les valeurs de $\mathrm{k}$ et de $\mathrm{C}_{\mathrm{g}} \mathrm{s}^{\prime}$ obtiennent à partir de l'expérience en traçant le graphe $\left[\mathrm{V}_{1 i \mathrm{~m}}, \ln \left(\mathrm{C}_{\bullet}\right)\right]$.

Du point de vue de la filtration, par application de la loi de Darcy, la vitesse de transfert de l'eau peut être reliée à la résistance hydraulique de la membrane $\mathrm{R}_{\mathrm{m}}$, par la relation :

$$
\mathrm{V}=\Delta \mathrm{P} / \mathrm{R}_{\mathrm{m}}
$$

En ultrafiltration, on rajoute à $\mathrm{R}_{\mathrm{m}}$ une résistance complémentaire $R_{g}$, pour tenir compte de l'effet de la polarisation et de la couche de gel :

$$
\mathrm{V}=\Delta \mathrm{P} /\left(\mathrm{R}_{\mathrm{m}}+\mathrm{R}_{\mathrm{g}}\right)
$$

Le modèle proposé pour la première fois par Goldsmith en 1971, fait intervenir la différence de pression osmotique des solutions de part et d'autre de la membrane. Cette pression a longtemps été ignorée car l'utilisation de l'équation de Van't Hoff conduit, pour les macromolécules, à des valeurs de $\Delta \pi$ négligeables devant $\Delta \mathrm{P}$. Or, plusieurs auteurs (Vilker et coll., 1981; Vink, 1971; Ogstrom et Preston, 1979) ont montré que la variation de pression osmotique avec la concentration obéit en réalité à une loi du type :

$$
\Delta \pi=\frac{\mathrm{R} \cdot \mathrm{T}}{\mathrm{M}} \cdot\left[\mathrm{C}+\mathrm{a} \cdot \mathrm{C}^{2}+\mathrm{b} \cdot \mathrm{C}^{3}+\ldots\right]
$$

Dans ces conditions, la vitesse de filtration est donnée par :

$$
\mathrm{V}=\mathrm{L} .(\Delta \mathrm{P}-\Delta \pi)
$$

$\mathrm{L}$ est la perméabilité hydraulique de la membrane.

Sa valeur est, en général, déterminée expérimentalement à partir de mesures de filtration d'eau à température constante $\left(L=1 / R_{m}\right)$. Par contre, la valeur de $\Delta \pi$ n'est accessible que si on connaît la concentration du soluté à la membrane. 
En utilisant la théorie du film et en tenant compte de l'influence de la pression osmotique, les relations (1), (5) et (6) permettent d'exprimer $\Delta \mathrm{P}$ en fonction de $\mathrm{V}$ :

$$
\Delta \mathrm{P}=\mathrm{V} \cdot \mathrm{R}_{\mathrm{m}}+\frac{\mathrm{R} \cdot \mathrm{T}}{\mathrm{M}}\left[\Sigma_{\mathrm{i}} \mathrm{C}_{\mathrm{i}} \cdot \exp \left(\frac{\mathrm{i} . \mathrm{V}}{\mathrm{k}}\right)\right]
$$

La résolution de cette équation nécessite la connaissance de la valeur de $\mathrm{k}$ qui peut être obtenue graphiquement dans la mesure où la relation (2) est vérifiée.

\section{DISPOSITIF EXPERIMENTAL ET METHODES UTILISEES}

Les manipulations d'ultrafiltration sont effectuées dans une cellule à agitation constante, et dont le volume est suffisant pour que l'on puisse négliger les variations de concentration en cours d'expérience (10\% au maximum). La membrane Rhône-Poulenc IRIS 3038 a la forme d'un disque de $17,4 \mathrm{~cm}^{2}$ d'aire utile et les solutions aqueuses sont constituées d'albumine bovine (Fraction v, IBF) à raison de 0,1 à $50 \mathrm{~g}$ par litre de solution. Le $\mathrm{pH}$ de ces solutions est ramené à 7,2 par addition de soude. La température est de $18^{\circ} \mathrm{C} \pm 1$ et les pressions relatives appliquées en cours d'ultrafiltration varient de 0 à $2.10^{5} \mathrm{~Pa}$.

Pour connaître l'influence du phénomène d'adsorption sur la résistance hydraulique de la membrane, la face active de cette dernière est mise en contact avec une solution protéique de concentration connue $C_{0}$, pendant une durée déterminée $t$, sous agitation $(15 \min <\mathrm{t}<15 \mathrm{~h})$. La membrane est ensuite rincée à l'eau distillée pour éliminer toute trace de solution d'imbibition. L'expérience montre qu'au bout d'un temps de rinçage de $15 \mathrm{~min}$ la perméabilité de la membrane n'est plus modifiée.

La variation de vitesse de filtration d'eau pure obtenue en appliquant diverses pressions suit la loi de Darcy (équation (3). On constate que la nouvelle résistance apparente de la membrane augmente en fonction du temps de contact entre la membrane et la solution et également en fonction de la concentration de cette dernière (fig. 1 et 2).

\section{RESULTATS}

Quelle que soit la concentration en BSA comprise entre 0,1 et $50 \mathrm{~g} / \mathrm{l}$, on constate que la résistance hydraulique de la membrane n'évolue plus au delà d'un temps de contact de 15 h (fig. 1). 


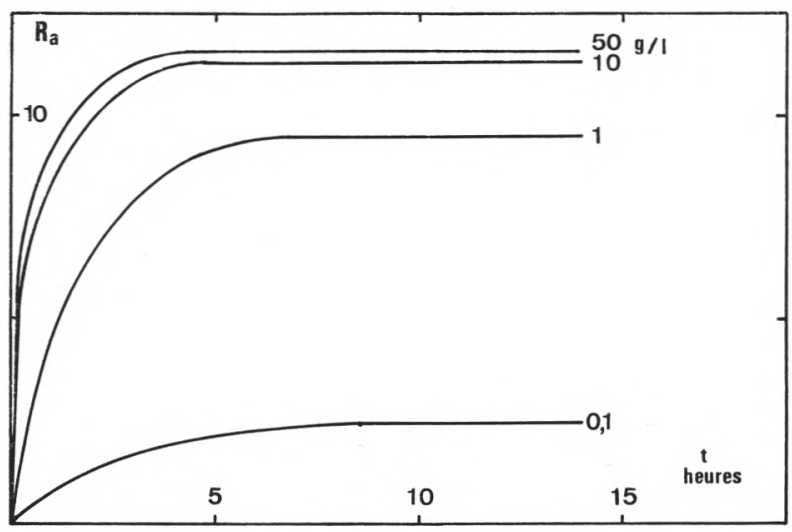

fig. 1

Résistance hydraulique de la membrane en fonction du temps d'adsorption $\mathrm{pH}=7,2, \mathrm{~T}=18^{\circ} \mathrm{C} \pm 1$ (équation 9).

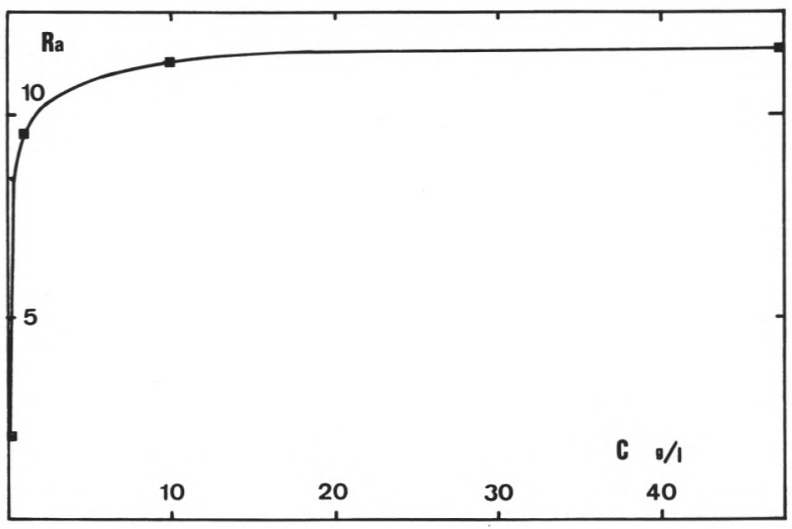

fig. 2

Résistance hydraulique de la membrane, après $15 \mathrm{~h}$ d'adsorption, en fonction de la concentration de $\mathrm{BSA}, \mathrm{pH}=7,2, \mathrm{~T}=18^{\circ} \mathrm{C} \pm 1$ (équation 8 ).

En traçant l'inverse de cette résistance en fonction de l'inverse de la concentration, on obtient une droite, ce qui permet de déduire la relation :

$$
R_{\mathrm{eq}}=\frac{50 . C}{1+4,25 . \mathrm{C}}
$$


Par ailleurs, à partir des courbes $\mathrm{R}=\mathrm{f}(\mathrm{t})$ établies à concentration constante pour des temps de contact variables, nous avons déterminé la loi d'évolution de la résistance au cours du temps :

$$
\mathrm{R}=\mathrm{R}_{\mathrm{m}}+\mathrm{A}\left(1-\exp \left(-\mathrm{C}^{0,3} \cdot \mathrm{t}\right)\right. \text { (9). }
$$

En combinant les équations (8) et (9), on obtient :

$$
R(C, t)=R_{m}+\frac{50 . C}{1+4,25 . C}\left(1-\exp \left(-C^{0,3} \cdot t\right)\right.
$$

Cette relation montre que la résistance de la membrane en fonctionnement est fortement influencée par l'adsorption, puisque $R_{\text {eq }}$ peut être de 3 à 4 fois supérieur à $R_{m}$.

Si on considère la membrane comme un milieu homogène percé de pores de diamètre $r$, on peut tenter d'interpréter l'adsorption d'un point de vue purement mécanique en supposant que les protéines adsorbées obstruent partiellement les pores qui ont alors un nouveau rayon équivalent $r-\Delta r$. En se fondant sur un modèle d'écoulement laminaire de l'eau dans le pore (Zeman, 1983) en accord avec la loi de Poiseuille, on peut exprimer la variation relative du rayon de pore au cours de l'adsorption en fonction des résistances $\mathrm{R}_{\mathrm{m}}$ et $\mathrm{R}_{\mathrm{eq}}$ :

$$
\frac{\Delta r}{r}=1-\left[{\frac{R_{m}}{R_{\text {eq }}}}^{0,25}\right]
$$

Sur la figure 3 nous avons représenté l'évolution de $\Delta \mathrm{r} / \mathrm{r}$ calculé d'après la relation (11) en fonction de la concentration $\mathrm{C}_{0}$ : il apparaît

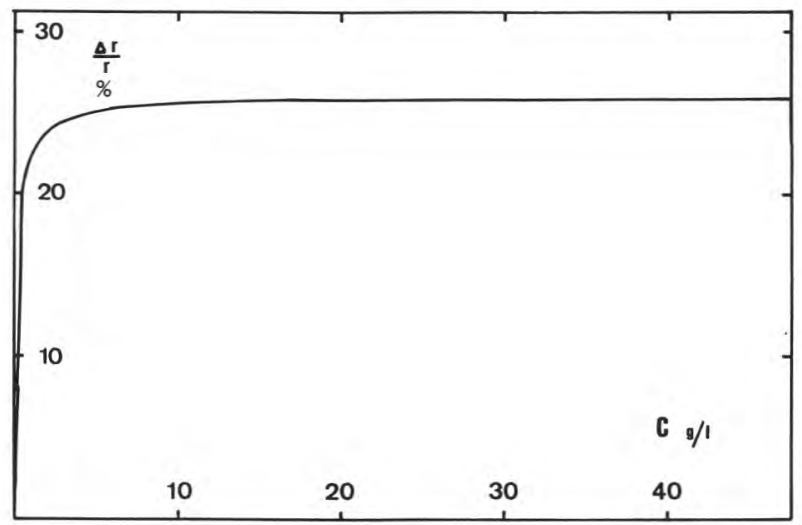

fig. 3

Variation relative du rayon de pore moyen d'une membrane après $15 \mathrm{~h}$ d'adsorption de $\mathrm{BSA}$ de concentration $\mathrm{C}_{0}$ variable $\mathrm{pH}=7,2, \mathrm{~T}=18^{\circ} \mathrm{C} \pm 1$ (équation 11 ). 
qu'une réduction de $30 \%$ de la section utile de passage peut être provoquée par la seule mise en contact de la membrane et de la solution. Cette remarque montre que s'il est important de connaître les caractéristiques intrinsèques de perméabilité hydraulique de la membrane, les interactions physicochimiques ne doivent pas être négligées si l'on veut approfondir la connaissance des phénomènes de transfert en ultrafiltration, par la recherche de modèles théoriques en particulier.

Par ailleurs, il est intéressant de remarquer que l'adsorption a une influence sur la forme des courbes $V=f(\Delta P)$ obtenues par ultrafiltration de solutions protéiques (fig. 4).

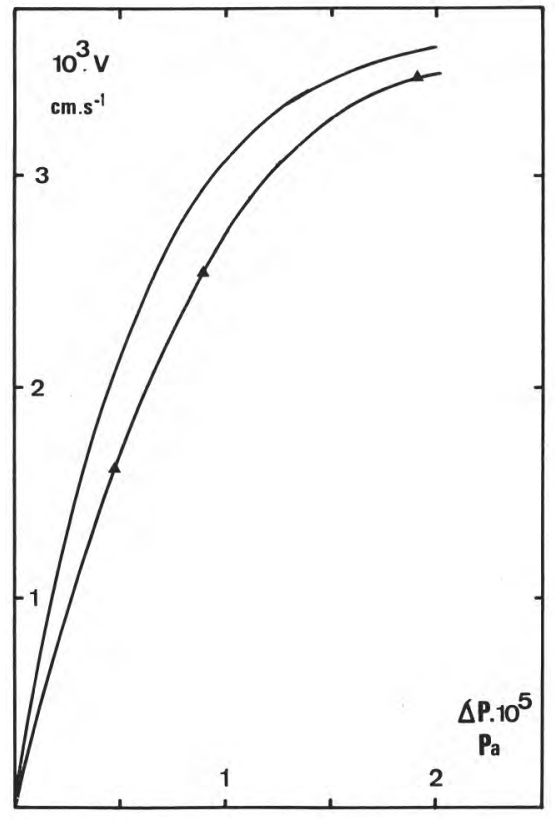

fig. 4

Vitesses de filtration expérimentales mesurées lors de l'ultrafiltration d'une solution de BSA. - membrane neuve, -\$- membrane " pré-adsorbée 》 $\mathrm{C}_{\mathrm{o}}=1 \mathrm{~g} / \mathrm{l}, \mathrm{pH}=7,2$.

C'est pour cette raison que les expériences d'ultrafiltration ont été menées après atteinte de l'équilibre d'adsorption : les résultats ainsi obtenus sont donnés sur la figure 5 . 


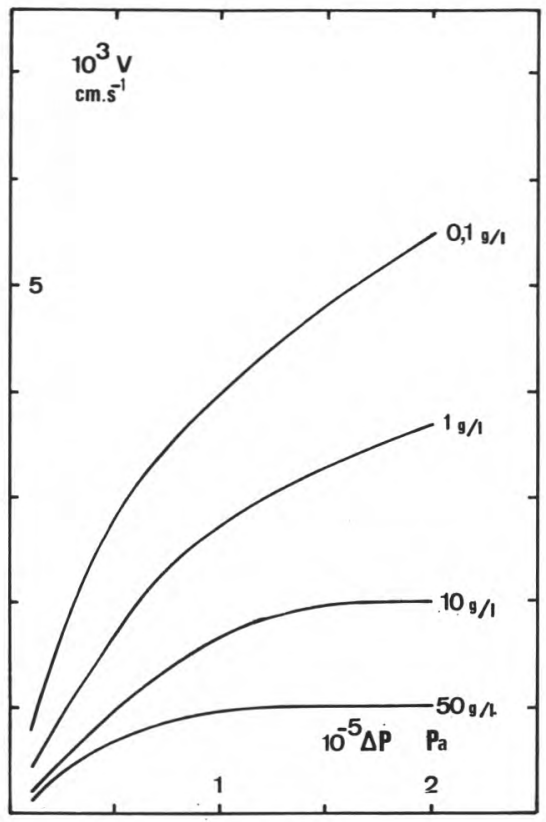

fig. 5

Vitesse de perméation en fonction de la différence de pression appliquée. La membrane est préalablement mise en présence de la solution à ultrafiltrer pendant $15 \mathrm{~h}, \mathrm{pH}=7,2$.

Afin d'interpréter ces courbes expérimentales, nous avons représenté sur les figures 6 et 7 les graphes de $(\mathrm{V}, \Delta \mathrm{P})$ calculés d'après l'équation (7), dans laquelle la résistance de la membrane est donnée successivement par les relations (3), (8) et (10). Ce n'est que dans ce dernier cas, faisant intervenir le temps de manipulation, que l'accord entre théorie et expérience est satisfaisant.

\section{DISCUSSION - CONCLUSION}

Ce travail montre l'importance de l'adsorption sur la résistance hydraulique des membranes, et met l'accent sur la nécessité de connaître l'état de celles-ci en cours de fonctionnement pour interpréter des résultats expérimentaux.

La simple mise en contact d'une membrane avec une solution de protéine, même de très faible concentration, provoque une modi- 


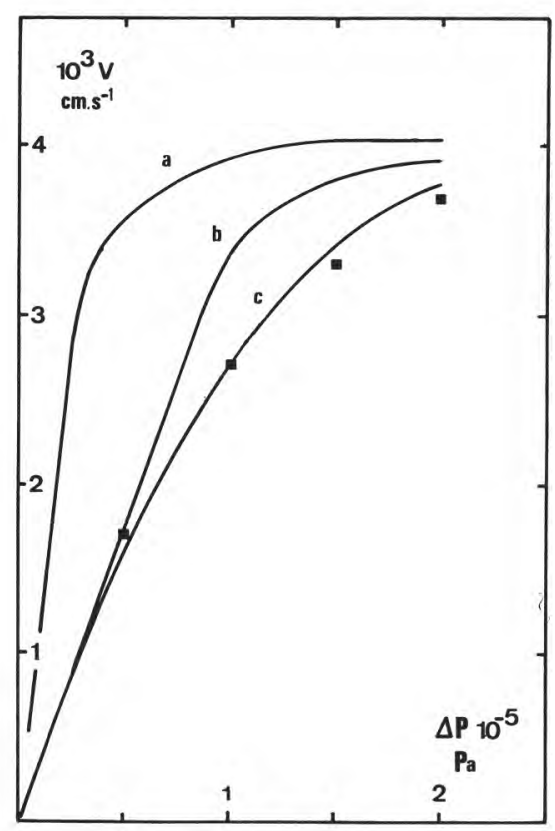

fig. 6

Comparaison entre les courbes $\mathrm{V}=\mathrm{f}(\Delta \mathrm{P})$ expérimentales et calculées.

- points expérimentaux.

a) équations ( 3 et 7 ), b) équations ( 8 et 7 ), c) équations (10 et 7 ) $\mathrm{C}_{0}=1 \mathrm{~g} / \mathrm{l}, \mathrm{pH}=7,2, \mathrm{~T}=18^{\circ} \mathrm{C} \pm 1$.

fication de sa perméabilité intrinsèque. Cette modification apparaît clairement lorsqu'on compare des courbes donnant la vitesse de filtration en fonction du temps à $\Delta \mathrm{P}$ et $\mathrm{C}_{0}$ constante, obtenues avec une membrane neuve et une membrane " pré-adsorbée " (fig. 8). Elle se comporte dès lors comme une membrane différente, dont le rayon de pore moyen calculé d'après la loi de Poiseulle, diminue. En conséquence, il paraît logique de prendre en compte ce phénomène lors de la caractérisation de la membrane par son taux de rejet et sa sélectivité.

L'importance de l'adsorption varie avec le couple (membranecomposition physico-chimique du milieu). Une étude analogue à celle qui est présentée ici devrait permettre de déterminer des prétraitements destinés à optimiser la compatibilité entre la membrane et la solution.

Par ailleurs, l'interprétation quantitative des courbes donnant la vitesse de filtration en fonction de la différence de pression appli- 


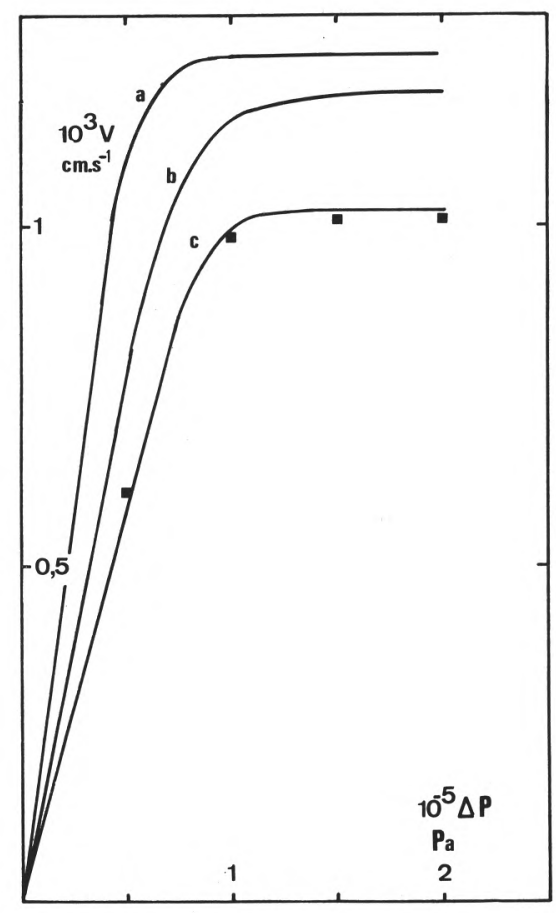

fig. 7

Comparaison entre les courbes $\mathrm{V}=\mathrm{f}(\Delta \mathrm{P})$ expérimentales et calculées.

- points expérimentaux.

a) équations ( 3 et 7$),$ b) équations ( 8 et 7 ), c) équations (10 et 7 ) $\mathrm{C}_{o}=50 \mathrm{~g} / \mathrm{l}, \mathrm{pH}=7,2, \mathrm{~T}=18^{\circ} \mathrm{C} \pm 1$.

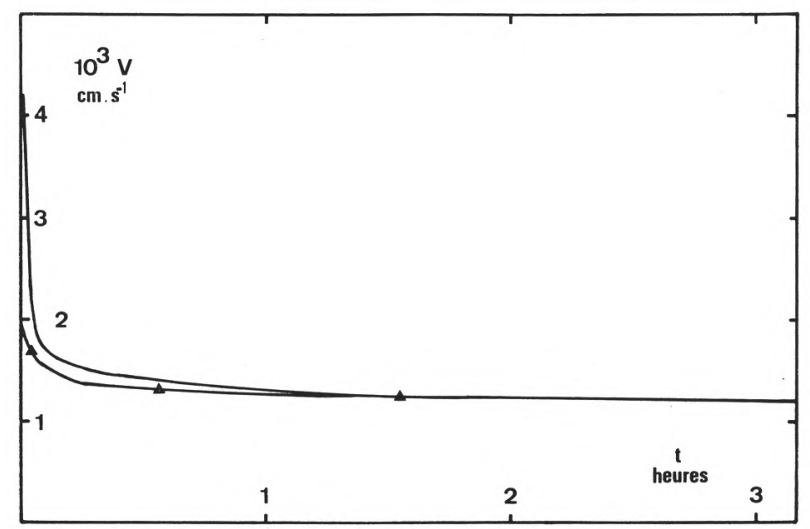

fig. 8

Variation de la vitesse de perméation en fonction du temps - membrane neuve, - $\mathbf{\Lambda}$ - membrane "pré-adsorbée " $\mathrm{C}_{0}=10 \mathrm{~g} / \mathrm{l}, \mathrm{pH}=7,2, \mathrm{~T}=18^{\circ} \mathrm{C} \pm 1, \mathrm{P}=10^{5} \mathrm{~Pa}$. 
quée, doit nécessairement prendre en compte l'influence de l'adsorption sur la résistance au transfert en fonction du temps et de la concentration. En effet, l'intégration dans les équations de transfert, de la loi $\mathrm{R}(\mathrm{C}, \mathrm{t})$, déterminée ici pour le couple (membrane IRISsolution de BSA) permet d'obtenir un accard satisfaisant entre les résultats calculés et expérimentaux. Ceci montre la nécessité d'expliciter les coefficients $\mathrm{L}$ de la théorie de la pression osmotique et $\mathrm{Rg}$ de la théorie de la filtration.

Enfin, l'utilisation de l'équation de transfert proposée $\Delta P=f(V$, $C, t)$, doit permettre, selon le point de fonctionnement choisi, de déterminer les importances relatives sur la limitation de la vitesse de filtration de l'adsorption, de la pression osmotique, de la polarisation, et éventuellement de la formation d'un gel.

\section{Bibliographie}

Blatt (W. F.), Dravid (A.), Michaels (A. S.), Nelsen (L.) (1970). - Solute polarization and cake formation in membrane ultrafiltration: causes, consequences, and control techniques. In J.E. Flinn (Ed.), Membrane science and technology. Plenum Press, p. 47-97.

FANE (A. G.), Fell (C. J.D.), SuKI (A.) (1983). - The effect of pH and ionic environment on the ultrafiltration of protein solutions with retentive membranes. J. of Membrane Sci., 16, 195-200.

GoLDSMITH (R. L.) (1971). - Macromolecular ultrafiltration with microporous membranes. Ind. Eng. Chem. Fundam., (10), 113-120.

Howell (J. A.). Velicangil (O.), Le (M. S.), Herrera Zeppelin (A. L.) (1981). Ultrafiltration of protein solutions. Annals of the N.Y. Acad. Sci., (369), 355-366.

Ingham (K. C.), Busby (T. F.), Sahlestrom (Y.), Castino (F.) (1980). - Separation of macromolecules by U.F. In A. R. Cooper (Ed.), Ultrafiltration membranes and applications, Plenum Press, N.Y., 141-158.

Kosinski (A. A.), Lightfoot (E. N.) (1972). - Protein ultrafiltration: A general example of boundary layer filtration. AICHE Journal, (18), 5, 1030-1041.

Ogston (A. G.), Preston (B. N.) (1979), - The molecular compression of Dextran. Biochem. J., (183), 1-9.

TRETTIN (D. R.), Doshi (M. R.) (1981), - Pressure independant ultrafiltration: is it gel limited or osmotic pressure limited. In A. F. Turbak (Ed.), Synthetic membranes, vol. 2, A.C.S. Symp. Series 154, A.C.S. Washington, p. 373.

VILKER (V. L.), COLTON (C. K.), SMITH (K. A.) (1981). - The osmotic pressure of concentrated protein solutions: Effect of concentration and $\mathrm{pH}$ in saline solutions of Bovine Serum Alb. J. Colloid. and Interface Sci. (79), 2, 548.

VINK (H.) (1971). - Precision measurements of osmotic pressure in concentrated polymer solutions. Europ. Polym. J., (7), 1411.

ZEMAN (L. J.) (1983). - Adsorption effects in rejection of macromolecules by ultrafiltration membranes. J. of Membrane Sci., (15), 3, 213-230. 